\title{
EXTENDING CONGRUENCE RELATIONS
}

\author{
PETER KRAUSS
}

\begin{abstract}
If $\mathfrak{A}$ and $\mathfrak{B}$ are algebras, where $\mathfrak{A} \subseteq \mathfrak{B}$, and $\theta$ is a congruence relation on $\mathfrak{A}$, let $\theta^{\mathfrak{B}}$ be the smallest congruence relation on $\mathfrak{B}$ containing $\theta . \mathfrak{A}$ is called a congruence subalgebra of $\mathfrak{B}$ if $\mathfrak{A} \subseteq \mathfrak{B}$ and, for every congruence relation $\theta$ on $\mathfrak{A}, \theta^{\mathfrak{B}} \cap|\mathfrak{U}|^{2}=\theta$. Elementary subalgebras are congruence subalgebras, and there are Directed Union and Loewenheim-Skolem Theorems for congruence subalgebras analogous to those for elementary subalgebras. Consequently we obtain full analogues of the Jónsson-Morley-Vaught results concerning homogeneous-universal algebras, where the notion of "subalgebra" is everywhere replaced by "congruence subalgebra".
\end{abstract}

In this note we shall consider algebras $\mathfrak{A}=\left\langle A, Q_{i}\right\rangle_{i_{\in} I}$ of similarity type $t \in \omega^{I}$ and the corresponding first order language $L_{t}$. Our notation and terminology follow a widely accepted pattern, and details can be found in Grätzer [4] and Morley and Vaught [9]. If $X$ is a set then $\kappa(X)$ is the power or cardinality of $X . \mathfrak{A}<\mathfrak{B}$ means that $\mathfrak{A}$ is an elementary subsystem of $\mathfrak{B}$. For later reference we state a version of the familiar "Robinson Lemmas" (see Robinson [10]).

LEMmA 1. If $\mathfrak{A}<\mathfrak{B}$ and, for each $k \in K, R_{k}^{\mathfrak{Q}}$ is a binary relation over $|\mathfrak{A}|$, then there exist $\mathfrak{C}$ and binary relations $R_{k}^{\mathfrak{C}}$ over $|\mathfrak{C}|$, for each $k \in K$, such that $\mathfrak{B}<\mathfrak{C}$ and $\left(\mathfrak{A}, R_{k}^{\mathfrak{2}}\right)_{k \in K}<\left(\mathfrak{C}, R_{k}^{\mathfrak{G}}\right)_{k \in K}$.

If $\mathfrak{A} \subseteq \mathfrak{B}$ and $\theta$ is a congruence relation on $\mathfrak{A}$, we denote by $\theta^{\mathfrak{B}}$ the smallest congruence relation on $\mathfrak{B}$ containing $\theta$. A characterization of $\theta^{\mathfrak{B}}$ can be found in Grätzer [4, p. 55]. $\mathfrak{A}$ is called a congruence subalgebra of $\mathfrak{B}$ (in symbols $\mathfrak{A} \triangleleft \mathfrak{B}$ ) if $\mathfrak{A} \subseteq \mathfrak{B}$ and, for every congruence relation $\theta$ on $\mathfrak{A}$, $\theta^{\mathfrak{B}} \cap|\mathfrak{A}|^{2}=\theta$.

We illustrate this notion with a few remarks. It is obvious that for Abelian groups $\mathfrak{A} \subseteq \mathfrak{B}$ if and only if $\mathfrak{A} \triangleleft \mathfrak{B}$. The same is true for distributive lattices and for Boolean algebras, as is well known. Congruence subalgebras play an essential role in the embedding theorems of Higman, Neumann

Received by the editors April 8, 1970 and, in revised form, March 31, 1971.

AMS 1969 subject classifications. Primary 5030.

Key words and phrases. Congruence relation, elementary subalgebra, Directed Union Theorem, Loewenheim-Skolem Theorem, embedding property, amalgamation property, homogeneous-universal algebra.

(c) American Mathematical Society 1972 
and Neumann [5] for groups, Evans [1], [2] for semigroups, quasigroups and loops, and Malcev [8] for rings, and many interesting examples can be found in these papers. In particular, for groups, semigroups, loops, rings and modular lattices it is easy to construct simple examples where $\mathfrak{A} \subseteq \mathfrak{B}$ but not $\mathfrak{A} \triangleleft \mathfrak{B}$. $^{1}$

The first two results have easy direct proofs using the well-known fact that $\mathfrak{A} \triangleleft \mathfrak{B}$ if and only if $\mathfrak{A} \subseteq \mathfrak{B}$ and $\theta^{\mathfrak{B}} \cap|\mathfrak{A}|^{2}=\theta$ for all finitely based congruence relations on $\mathfrak{A}$.

THEOREM 2. Suppose $\left\{\mathfrak{H}_{i} \mid i \in I\right\}$ is a $\subseteq$-directed family, and $\mathfrak{A}_{i} \triangleleft \mathfrak{B}$ for all $i \in I$. Then $\bigcup\left\{\mathfrak{A}_{i} \mid i \in I\right\} \triangleleft \mathfrak{B}$.

THEOREM 3. Suppose $\left\{\mathfrak{A}_{i} \mid i \in I\right\}$ is a $\triangleleft$-directed family. Then $\mathfrak{A}_{j} \triangleleft$ $\bigcup\left\{\mathfrak{Q}_{i} \mid i \in I\right\}$ for all $j \in I$.

The main observation of this note is stated next.

THEOREM 4. If $\mathfrak{A}<\mathfrak{B}$, then $\mathfrak{A} \triangleleft \mathfrak{B}$.

PRoof. By Lemma 1 , there exists $\mathfrak{C}$ and binary relations $R_{\theta}$ over $|\mathfrak{C}|$, for each congruence relation $\theta$ on $\mathfrak{A}$, such that $\mathfrak{B}<\mathfrak{C}$ and $(\mathfrak{A}, \theta)_{\theta \in \Theta(\mathfrak{I})}<$ $\left(\mathfrak{C}, R_{\theta}\right)_{\theta \in \Theta(\mathfrak{O})}$, where $\Theta(\mathfrak{U})$ is the set of all congruence relations on $\mathfrak{U}$. Since to be a congruence relation on $\mathfrak{A}$ is an elementary property, $\boldsymbol{R}_{\boldsymbol{\theta}}$ is a congruence relation on $\mathfrak{C}$, for all $\theta \in \Theta(\mathfrak{H})$. Also $R_{\theta} \cap|\mathfrak{A}|^{2}=\theta$, and therefore $\theta \subseteq \theta^{\mathfrak{C}} \cap|\mathfrak{M}|^{2} \subseteq R_{\theta} \cap|\mathfrak{M}|^{2}=\theta$, for all $\theta \in \Theta(\mathfrak{H})$. Thus $\mathfrak{A} \triangleleft \mathfrak{C}$, and since $\mathfrak{A} \subseteq \mathfrak{B} \subseteq \mathfrak{C}, \mathfrak{A} \triangleleft \mathfrak{B}$.

Theorem 4 has important consequences, such as an analogue of the "downward" Loewenheim-Skolem Theorem for the relation $\triangleleft$.

CoROllary 5. Let $\kappa(\mathfrak{B}) \geqq \kappa \geqq \omega, \kappa(I)$, and $X \subseteq|\mathfrak{B}|$ such that $\kappa(X) \leqq \kappa$. Then there exists $\mathfrak{A} \triangleleft \mathfrak{B}$ such that $\kappa(\mathfrak{H})=\kappa$ and $X \subseteq|\mathfrak{A}|$.

Proof. Theorem 4 and the "downward" Loewenheim-Skolem Theorem.

Similarly, of course, we have an "upward" version of the LoewenheimSkolem Theorem for the relation $\triangleleft$, and there are other useful applications of theorems from model theory.

It is now easy to see (and known from category theory-see, e.g. Fleischer [3]) that with the obvious modifications of the embedding and amalgamation properties, we obtain full analogues of the JónssonMorley-Vaught results concerning special and homogeneous-universal algebras (see Jónsson [6], [7] and Morley and Vaught [9]), where the notion of "subalgebra" is everywhere replaced by "congruence subalgebra".

\footnotetext{
${ }^{1}$ In Evans [1] it is claimed that for quasigroups and loops $\mathfrak{A} \subseteq \mathfrak{B}$ if and only if $\mathfrak{A} \triangleleft \mathfrak{B}$. However this claim was retracted in Evans [2].
} 
Using elementary properties of direct products, free products and free products with amalgamated subalgebras, the reader will quickly verify the following observations.

LeMma 6. If $\mathscr{M}$ is closed under direct products, and every member of $\mathscr{M}$ has a one-element subalgebra, then $\mathscr{M}$ has the congruence embedding property.

Lemma 7. If $\mathscr{M}$ is an equational class, then $\mathscr{M}$ has the embedding property if and only if $\mathscr{M}$ has the congruence embedding property.

LeMma 8. If $\mathscr{M}$ is an equational class with the embedding and amalgamation properties, then $\mathscr{M}$ has the congruence amalgamation property.

It follows that many classes $\mathscr{M}$ of algebras, for which the existence of $\mathscr{M}$-homogeneous-universal algebras has been established, have $\mathscr{M}$ congruence homogeneous-universal algebras subject to exactly the same cardinality restrictions. To mention only a few examples, under the assumption of the generalized continuum hypothesis there exists, for each uncountable regular cardinal $\kappa$, up to isomorphism exactly one congruence homogeneous-universal group, Abelian group, lattice, distributive lattice, Boolean algebra, etc., of cardinality $\kappa$.

In view of Lemma 8, an interesting problem is to consider classes of algebras which have the embedding property but fail to have the amalgamation property, such as semigroups and rings. The author has not been able to settle the question whether these classes have the congruence amalgamation property.

It is clear that an $\mathscr{M}$-homogeneous algebra is $\mathscr{M}$-congruence homogeneous, whereas an $\mathscr{M}$-congruence universal algebra is $\mathscr{M}$-universal. Thus a strengthening of universality has been obtained at the expense of homogeneity. On the other hand we gain on homogeneity for homomorphisms, as a straightforward argument establishes our next result.

THEOREM 9. Let $\kappa$ be an infinite cardinal. Suppose $\mathscr{M}$ is closed under homomorphisms and unions of $\subseteq$-chains, and for every $\mathfrak{A} \in \mathscr{M}$ and $X \subseteq|\mathfrak{A}|$, where $\kappa(X)<\kappa$, there exists $\mathfrak{B} \triangleleft \mathfrak{A}$, where $\mathfrak{B} \in \mathscr{M}$ and $\kappa(\mathfrak{B})<\kappa$, such that $X \subseteq|\mathfrak{B}|$. Let $\mathfrak{A}$ be $\mathscr{M}$-congruence homogeneous-universal, where $\kappa(\mathfrak{H})=\kappa$. Then for any $\mathfrak{B}, \mathfrak{B}^{\prime} \triangleleft \mathfrak{A}$, where $\mathfrak{B}, \mathfrak{B}^{\prime} \in \mathscr{M}$ and $\kappa(\mathfrak{B}), \kappa\left(\mathfrak{B}^{\prime}\right)<\kappa$, and any homomorphism f from $\mathfrak{B}$ onto $\mathfrak{B}^{\prime}$, there exists $\mathfrak{C} \triangleleft \mathfrak{A}$, where $\mathfrak{C} \in \mathscr{M}$, and a homomorphism $g$ of $\mathfrak{A}$ onto $\mathfrak{C}$ such that $f \subseteq g$.

Notice that, by Theorem 4, the third ("downward" LoewenheimSkolem) part of the hypothesis of Theorem 9 is satisfied whenever $\mathscr{M} \in E C_{\Delta}$ (elementary class in the wider sense) and $\omega, \kappa(I)<\kappa$. Therefore 
the conclusion of Theorem 9 applies to many interesting classes of algebras such as, e.g., all equational classes.

We conclude with an application of our results to elementary types. First we state an immediate consequence of Morley and Vaught $[9,2.4]$ and Theorem 4.

TheOREm 10. Suppose $\mathscr{N} \in E T$, and $\mathscr{N}$ contains infinite algebras. Then every special algebra $\mathfrak{A} \in \mathscr{N}$ is $\mathscr{N}$-congruence universal.

With the help of Theorem 10 we then obtain an analogue of Morley and Vaught $[9,4.1]$ for $\mathscr{M}$-congruence universal algebras.

THEOREM 11. Assume the generalized continuum hypothesis. If $\mathscr{M} \in \boldsymbol{E} \boldsymbol{C}_{\Delta}$ and $\omega, \kappa(I)<\kappa$, then there exists an $\mathscr{M}$-congruence universal algebra of power $\kappa$ if and only if $\mathscr{M}$ has the congruence embedding property.

From Lemma 7 we conclude that under the assumption of the generalized continuum hypothesis there exists, for each uncountable cardinal $\kappa$, a congruence universal semigroup and ring of cardinality $\kappa$.

\section{REFERENCES}

1. T. Evans, Embedding theorems for multiplicative systems and projective geometries, Proc. Amer. Math. Soc. 3 (1952), 614-620. MR 14, 347.

2. - On multiplicative systems defined by generators and relations. II, Proc. Cambridge Philos. Soc. 49 (1953), 579-589. MR 15, 283.

3. I. Fleischer, A note on universal homogeneous models, Math. Scand. 19 (1966), 183-184. MR 35 \#4095.

4. G. Grätzer, Universal algebra, Van Nostrand, Princeton, N.J., 1968. MR 40 \#1320.

5. G. Higman, B. H. Neumann and H. Neumann, Embedding theorems for groups, J. London Math. Soc. 24 (1949), 247-254. MR 11, 322.

6. B. Jónsson, Universal relational systems, Math. Scand. 4 (1956), 193-208. MR 20 \#3091.

7. - Homogeneous universal relational systems, Math. Scand. 8 (1960), 137-142. MR 23 \#A2328.

8. A. I. Mal'cev, On a representation of nonassociative rings, Uspehi Mat. Nauk 7 (1952), no. 1 (47), 181-185. MR 13, 816.

9. M. Morley and R. Vaught, Homogeneous universal models, Math. Scand. 11 (1962), 37-57. MR 27 \#37.

10. A. Robinson, $A$ result on consistency and its application to the theory of definition, Nederl. Akad. Wetensch. Proc. Ser. A. 59=Indag. Math. 18 (1956), 47-58. MR 17, 1172.

Department of Mathematics, Emory University, Atlanta, Georgia 30322

Department of Mathematics, State University of New York, New Paltz, NEW YORK 12561

Current address: Department of Mathematics, University of Heidelberg, Heidelberg, West Germany 